\title{
EPSO - BEST-OF-TWO-WORLDS META-HEURISTIC APPLIED TO POWER SYSTEM PROBLEMS
}

\author{
Vladimiro Miranda \\ vmiranda@inescporto.pt_nfonseca@inescporto.pt \\ INESC Porto - Instituto de Engenharia de Sistemas e Computadores do Porto, Portugal \\ \& FEUP - Faculdade de Engenharia da Universidade do Porto, Portugal
}

\begin{abstract}
This paper presents a new meta-heuristic (EPSO) built putting together the best features of Evolution Strategies (ES) and Particle Swarm Optimization (PSO). Examples of the superiority of EPSO over classical PSO are reported. The paper also describes the application of EPSO to real world problems, including an application in Opto-electronics and another in Power Systems.
\end{abstract}

Keywords - Evolution Strategies, Evolutionary Programming, Particle Swarm Optimization

\section{INTRODUCTION}

This paper deals with a new variant in the family of metaheuristic algorithms, built over the concepts of Evolution Strategies (or Evolutionary Programming) and of Particle Swarm Optimization. It is only fair that the method is called EPSO - Evolutionary Particle Swarm Optimization.

Under the name of Evolution Strategies (ES) - see [1] and [2] - and Evolutionary Programming (EP) - see [3] - a number of models have been developed that rely on Darwinist selection to promote progress towards the (unknown) optimum. This selection procedure may rely on pure stochastic basis or have a deterministic flavor, include elitism or yet other characteristics - but, in the end, the general principle of the "survival of the fittest" remains.

These models all use a phenotypic representation of the solutions of a problem, contrary to Genetic Algorithms, where a binary chromosome is adopted instead. Also, as an inductor of variation, they use processes like mutation and recombination.

The ES approach to optimization has proven successful in many areas, allowing one to obtain solutions of high quality for very difficult problems. However, ES models may be time consuming and experiment difficulties for very large problems and therefore anything that may accelerate convergence will be seen as an advance.

The most modern ES models combine competition with selfadaptation. Not only individuals are evolving towards the optimum but also some of the characteristics that command their evolution are also evolving, making the whole process "learn" about the best way to achieve progress.

Particle Swarm Optimization algorithms [4] [5] rely on a different concept. Mimicking zoological behavior, imitating the collective or social behavior of animal swarms, flocks or schools, a set of particles evolves in the search space motivated by three factors: habit, memory and cooperation.

The first factor impels a particle to follow a path along its previous movement direction (it is often called the inertia factor). The second factor influences the particle to come back on its steps, i.e., to tend to go back to the best position it found during its life. The third factor (information exchange) induces the particle to move closer to the best point in space found by the collective of all particles in its family or group.

In classical PSO there is no competition among particles or self-adaptation of characteristics. In fact, if it were not the information exchange factor, each particle would evolve independently of all the others. It is this cooperation factor that gives all the power to the PSO model. PSO is a recent paradigm but it has already demonstrated a big potential, although it seems sensitive to the tuning of some weights or parameters.

In this paper, we introduce a third view: Evolutionary selfadapting Particle Swarm Optimization (EPSO). EPSO puts together concepts of ES and of PSO. We will have a swarm of particles evolving in the search space; however, these particles will also be subject to selection under the evolutionary paradigm. This selection acts on the weights or parameters governing the behavior of a particle and, therefore, one might say that particles displaying the fittest behavior will survive and propagate.

We, however, prefer to explain the method from an evolutionary point of view - we see EPSO as an evolutionary model where a new operator is introduced to generate diversity: the particle movement operator, which contributes to the increase of the value of the progress rate towards the optimum.

Using the vocabulary of ES, we have therefore a swarm of particles that have object parameters (the variables of the problem) and strategic parameters (the parameters that define the relative weight of the control factors in particle swarm movement); the object parameters evolve according to the swarm behavior rules, but the strategic parameters are selected according to a self-adaptive evolution strategy procedure. Therefore, we expect to join together the exploratory power of PSO with the self-adaptation power of ES and have as a result the "best of two worlds".

As we will demonstrate, EPSO is a very successful model. However, it is only fair to give credit to other attempts to build bridges between PSO and the world of Evolutionary Computing, such as in [6], or to give an adaptive flavor to a Swarm-type algorithm, such as in [7]. 


\section{BRIEF REVIEW OF CLASSICAL PSO}

In the classical PSO, one must have, at a given iteration, a set of solutions or alternatives called "particles". From one iteration to the following, each particle $\mathbf{X}_{\mathrm{i}}$ moves according to a rule that depends on three factors, as follows.

In order to understand this rule, one must also keep record of the best point $\mathbf{b}_{\mathrm{i}}$ found by the particle in its past life and the current global best point $\mathbf{b}_{\mathrm{g}}$ found by the swarm of particles in their past life.

The movement rule states that

$$
\mathbf{X}_{\mathrm{i}}^{\text {new }}=\mathbf{X}_{\mathrm{i}}+\mathbf{V}_{\mathrm{i}}^{\text {new }}
$$

where $V_{i}$ is called the particle $i$ velocity and is defined by

$\mathbf{V}_{\mathrm{i}}^{\text {new }}=\operatorname{Dec}(\mathrm{t}) \mathrm{w}_{\mathrm{i} 0} \mathbf{V}_{\mathrm{i}}+\operatorname{Rnd}_{1} \mathrm{w}_{\mathrm{i} 1}\left(\mathbf{b}_{\mathrm{i}}-\mathbf{X}_{\mathrm{i}}\right)+\operatorname{Rnd}_{2} \mathrm{w}_{\mathrm{i} 2}\left(\mathbf{b}_{\mathrm{g}}-\mathbf{X}_{\mathrm{i}}\right)$

where the first term of the summation represents inertia or habit (the particle keeps moving in the direction it had previously moved), the second represents memory (the particle is attracted to the best point in its trajectory) and the third represents cooperation or information exchange (the particle is attracted to the best point found by all particles).

The parameters $\mathrm{w}_{\mathrm{ik}}$ are weights fixed in the beginning of the process. $\mathrm{Rnd}_{\mathrm{x}}$ are random numbers sampled from a uniform distribution in $[0,1]$. Dec(t) is a function decreasing with the progress of iterations, reducing progressively the importance

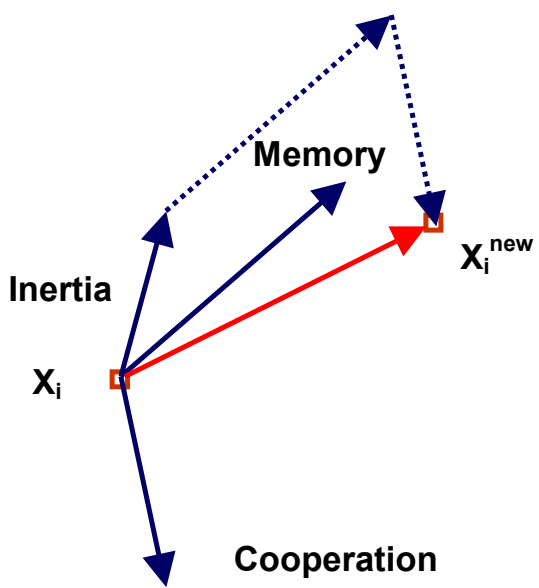

of the inertia term [8]. Figure 1 illustrates the concept.

Figure 1 - Illustrating the movement of a particle, influenced by three terms.

\section{BRIEF REVIEW OF EVOLUTION STRATEGIES}

In the $\sigma$ SA-ES (self-adaptive Evolution Strategies) model, one must have, at a given iteration called generation, a set of solutions or alternatives called individuals. Each individual is characterized by object parameters (the values of the variables describing a solution) and by strategic parameters (mutation rates for each variable, mutation correlation angles and similar).
Although there are many variants, we will describe the following procedure for reasons that will become obvious later on:

- $\quad$ Each individual is duplicated

- The strategic parameters of each individual undergo mutation

- The object parameters of each individual are mutated under a procedure commanded by its strategic parameters (this generates new individuals)

- A number of individuals undergo recombination (this generates new individuals)

- From the set of parents and sons (the original and the new individuals), the best fit are selected to form a new generation

The selection procedure has a number of variants and can be ruled by a stochastic tournament or be purely deterministic, involve elitism, niching, etc.

There is an interesting theoretical building providing insight on why ES achieve convergence and how a near optimal progress rate is achieved [9].

\section{CRITICIZING PSO}

The most striking point of classical PSO is the fact that it depends of a number of parameters defined externally by a user, and most certainly with values that are problem dependent.

This is certainly true for the definition of the weights $\mathrm{w}_{\mathrm{ik}}$, and our experience seems to be in agreement with other authors: a delicate work of tuning the algorithm is often necessary, in practical problems.

Furthermore, the external definition of the decreasing function $\operatorname{Dec}(\mathrm{t})$ is also something that can only leave on with a feeling of discomfort. It is intuitive that if the inertia term is eliminated at an early stage of the process, then the algorithm risks to be trapped at some local minimum. Therefore, some more tuning is needed.

To avoid this kind of problem, some authors have suggested procedures of "re-seeding" the search by generating new particles at distinct places of the search space.

Last, the random factors $\mathrm{Rnd}_{\mathrm{k}}$, while introducing an useful stochastic flavor, only have a heuristic basis and are not sensitive to the evolution of the process.

Observing PSO as an proto-evolutionary process, we may say that:

- We have in PSO a mechanism to reproduce and generate new individuals from a previous set (the movement rule) - i.e., we see the new iteration not as a movement of particles but as a generation of new alternatives in different positions in space

- But we do not have an explicit selection mechanism in 
the Darwinist sense; however, the algorithm exhibits a positive progress rate because the movement rule induces such property implicitly.

\section{EPSO -EVOLUTIONARY SELF-ADAPTING PSO}

The idea behind EPSO [10] is to grant a PSO scheme with an explicit selection procedure and with self-adapting properties for its parameters.

At a given iteration, consider a set of solutions or alternatives that we will keep calling particles. The general scheme of EPSO is the following:

- $\quad$ REPLICATION - each particle is replicated $r$ times

- $\quad$ MUTATION - each particle has its weights mutated

- $\quad$ REPRODUCTION - each mutated particle generates an offspring according to the particle movement rule

- $\quad$ EVALUATION - each offspring has its fitness evaluated

- SELECTION - by stochastic tournament the best particles survive to form a new generation

The movement rule for EPSO is the following: given a particle $\mathbf{X}_{i}$, a new particle $\mathbf{X}_{i}^{\text {new }}$ results from

$$
\begin{gathered}
\mathbf{X}_{\mathrm{i}}^{\text {new }}=\mathbf{X}_{\mathrm{i}}+\mathbf{V}_{\mathrm{i}}^{\text {new }} \\
\mathbf{V}_{\mathrm{i}}^{\text {new }}=\mathrm{w}_{\mathrm{i} 0}^{*} \mathbf{V}_{\mathrm{i}}+\mathrm{w}_{\mathrm{i} 1}^{*}\left(\mathbf{b}_{\mathrm{i}}-\mathbf{X}_{\mathrm{i}}\right)+\mathrm{w}_{\mathrm{i} 2}^{*}\left(\mathbf{b}_{\mathrm{g}}^{*}-\mathbf{X}_{\mathrm{i}}\right)
\end{gathered}
$$

So far, this seems like PSO - the movement rule keeps its terms of inertia, memory and cooperation. However, the weights undego mutation

$$
\mathrm{w}_{\mathrm{ik}}^{*}=\mathrm{w}_{\mathrm{ik}}+\tau \mathrm{N}(0,1)
$$

where $\mathrm{N}(0,1)$ is a random variable with Gaussian distribution, 0 mean and variance 1 ; and the global best $\mathbf{b}_{\mathrm{g}}$ is randomly disturbed to give

$$
\mathbf{b}_{\mathrm{g}}^{*}=\mathbf{b}_{\mathrm{g}}+\tau^{\prime} \mathrm{N}(0,1)
$$

The $\tau, \tau$, are learning parameters (either fixed or treated also as strategic parameters and therefore also subject to mutation).

This scheme benefits from two "pushes" in the right direction: the Darwinistic process of selection and the particle movement rule and therefore it is natural to expect that it may display advantageous convergence properties when compared to ES or PSO alone.

Furthermore, EPSO can also be classified as a self-adaptive algorithm, because it relies on the mutation and selection of strategic parameters, just as any $\sigma$-SA Evolution Strategy.

\section{EXPERIENCE WITH EPSO}

We have conducted a large number of experiments that have convinced us of the superiority of EPSO. Some of these experiments have been performed on classical test problems and in this section we will report results for some of them.

\subsection{Test functions}

The two first functions represent difficult optima to be found, the third is the classical function that was used by ES researchers to define the concepts of progress rate [9] and the forth exhibits a large number of deceiving local optima.

$\underline{\text { Schaffer's function: }}$

$$
f_{1}(x)=0.5+\frac{\left(\sin \sqrt{x^{2}+y^{2}}\right)^{2}-0.5}{\left(1.0+0.001\left(x^{2}+y^{2}\right)\right)^{2}}
$$

$\underline{\text { Rosenbrock function: }}$

$$
f_{2}(x)=\sum_{i=1}^{n}\left(100 \times\left(x_{i+1}-x_{i}^{2}\right)^{2}+\left(x_{i}-1\right)^{2}\right)
$$

Sphere function:

$$
f_{3}(x)=\sum_{i=1}^{n} x_{i}^{2}
$$

Alpine function:

$$
\mathrm{f}_{4}(\mathbf{X})=\sin \left(\mathrm{x}_{1}\right) \ldots \sin \left(\mathrm{x}_{\mathrm{n}}\right) \times \sqrt[n]{\mathrm{x}_{1} \ldots \mathrm{x}_{\mathrm{n}}}
$$

The parameters used in the tests are presented in Table 1. The threshold used as the stopping criterion is listed in the "Stop" column (a value close to the exact optimum).

\begin{tabular}{||c|c|c|c||}
\hline \hline Function & $\mathrm{n}$ & Domain $^{\mathrm{n}}$ & Stop \\
\hline$f_{1}$ & 2 & {$[-50,50]^{\mathrm{n}}$} & $1.0 \mathrm{E}-10$ \\
\hline$f_{2}$ & 30 & {$[0,30]^{\mathrm{n}}$} & 100 \\
\hline$f_{3}$ & 30 & {$[-50,50]^{\mathrm{n}}$} & 0.01 \\
\hline$f_{4}$ & 2 & {$[0,100]^{\mathrm{n}}$} & 98.9627 \\
\hline
\end{tabular}

Table 1: Parameters used in the test functions.

\subsection{Results in the test functions}

The following pictures illustrate the typical convergence in the test functions, for the EPSO and PSO. The graphs display the evolution of the value of the best solution along the successive iterations, and the comparison is made in terms of "number of evaluations", and not in number of iteration steps, so that a more fair computing effort comparison can be made.

The EPSO trials were done by setting the replication index $r$ equal to 2 (a particle give origin to two descendents, one of them mutated in its strategic parameters, and this offspring is subject to stochastic tournament selection). PSO requires only one evaluation per particle per iteration, but EPS requires $r$ evaluations per particle per iteration.

The results presented were obtained with a population of 20 particles, over 500 simulations. 


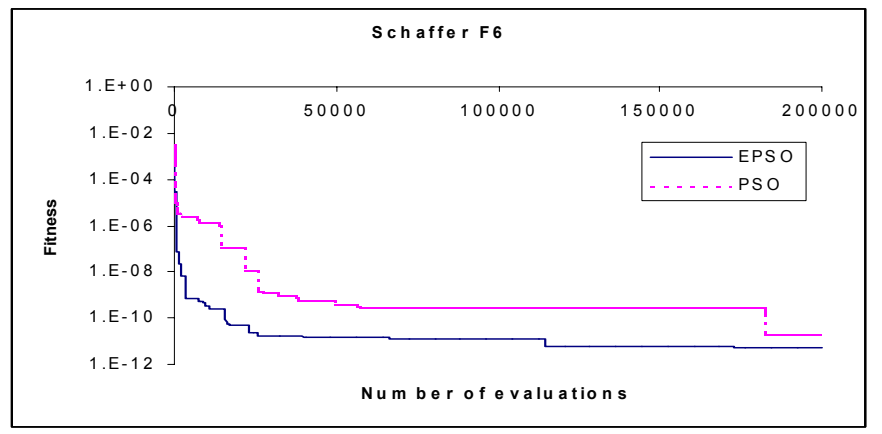

Figure 1 - Typical convergence in the Schaffer's function.

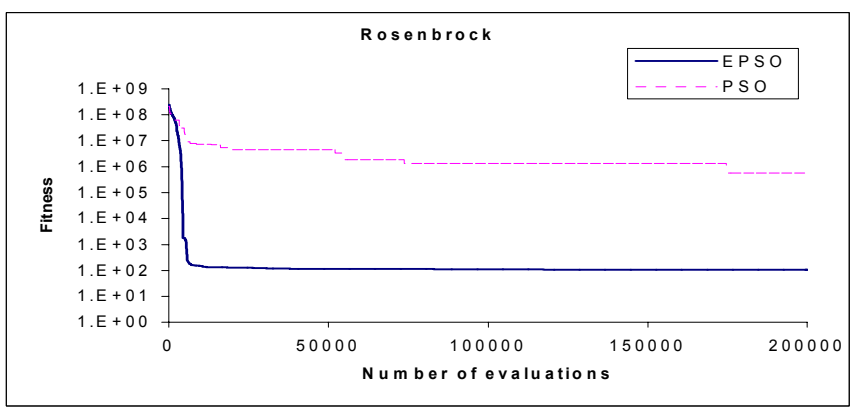

Figure 2 - Typical convergence in the Rosenbrock function.

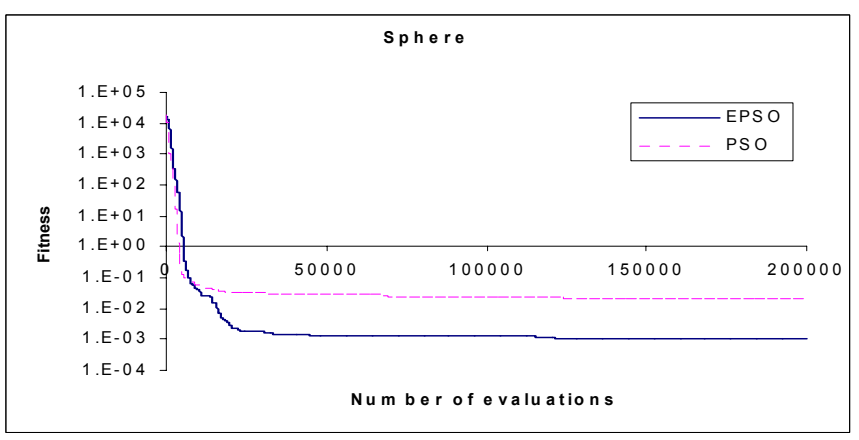

Figure 3 - Typical convergence in the Sphere function.

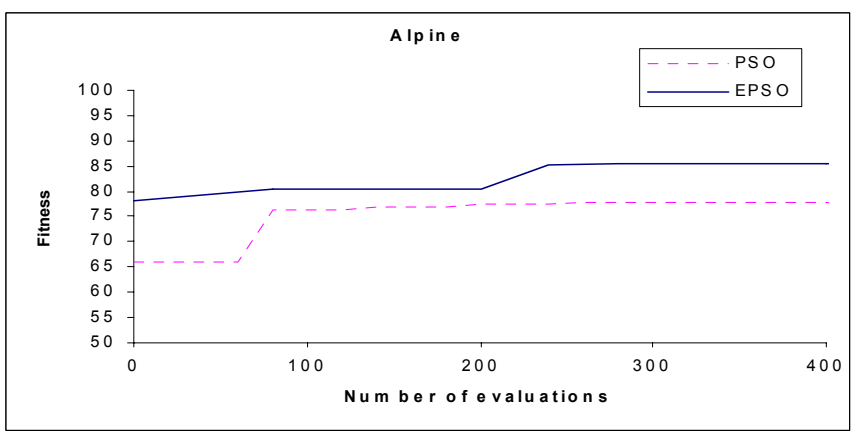

Figure 4 - Typical convergence in the Alpine function.

The results presented in Figures 1, 2, 3 and 4 show a clear superiority of the EPSO algorithm. The PSO results could perhaps be optimized if we'd invested an extra effort in tuning by hand the strategic parameters. EPSO was able to provide better results independently of the strategic parameter initialization.
Notice that the position and the strategic parameters (inertia, coordination factor and memory) were always initialized randomly for each particle. The results of the PSO would have perhaps a margin for improvement if the strategic parameters were tuned. EPSO algorithm was able to provide good results independently of that initialization.

This is a very important improvement in the algorithm, because the results of Classic PSO are very dependent of the strategic parameter initialization [8].

\subsection{MIN-MAX FITNESS FUNCTIONS}

All the previous examples were based on differentiable objective functions of continuous variables.

However, in practice one meets other types of problems including discrete variables, or other type of objective functions. For instance, in Power System planning, there are problems formulated with objective functions of the min-max type - for instance, in Risk Analysis. Therefore, it is interesting to verify if EPSO behaves well for such problems.

We have solved, with EPSO, a problem in the design of fiber optics filters built with Bragg gratings technology [11]. A Bragg grating is a periodic modulation of the index of refraction along the fiber core. We will not enter in the details of this problem, but we may summarize it as follows:

- A Bragg grating optical filter may be produced by controlled laser beams causing a series of "markings" in the glass fiber

- The number of these markings and their mutual distance influence the way light is reflected, when a polychromatic wave travels inside the fiber

- A frequency filter is ideal when it reflects only a specific target frequency and allows all other frequencies to travel through without attenuation

There are mathematical models known in Physics that, given the geometry of a grating, will tell what's the behavior of the filter in terms of frequencies reflected and attenuated. However, there is no direct answer to the inverse problem: how to design a grating that may display a specific filtering behavior.

This seemed the perfect problem to test EPSO. It includes continuous variables (distance between two consecutive gratings) and discrete variables (number of sections with distinct grating distances, and number of gratings within each section). Furthermore, the objective function was of the minMax type:

$$
\min \left[\operatorname{Max}\left\{\text { Reflection } \text { error }_{\mathrm{I}}\right\}, \mathrm{i} \in \mathrm{F}\right]
$$

where

$\mathrm{F}$ - set of frequencies around the target frequency Reflection $_{\text {error }}{ }_{\mathrm{i}}=$ Desired Reflection $_{\mathrm{i}}-$ Solution Reflection $_{\mathrm{i}}$

This problem was successively solved by EPSO and has provided INESC with a tool to manufacture optical filters according to design specifications. Without further details, we show in Figure 5 a graph of the reflection spectrum of a 
complex Bragg grating as a function of wavelength, its design solution obtained by an EPSO model. It goes without saying that this reflection spectrum was considered as of high quality by the optical communication experts.

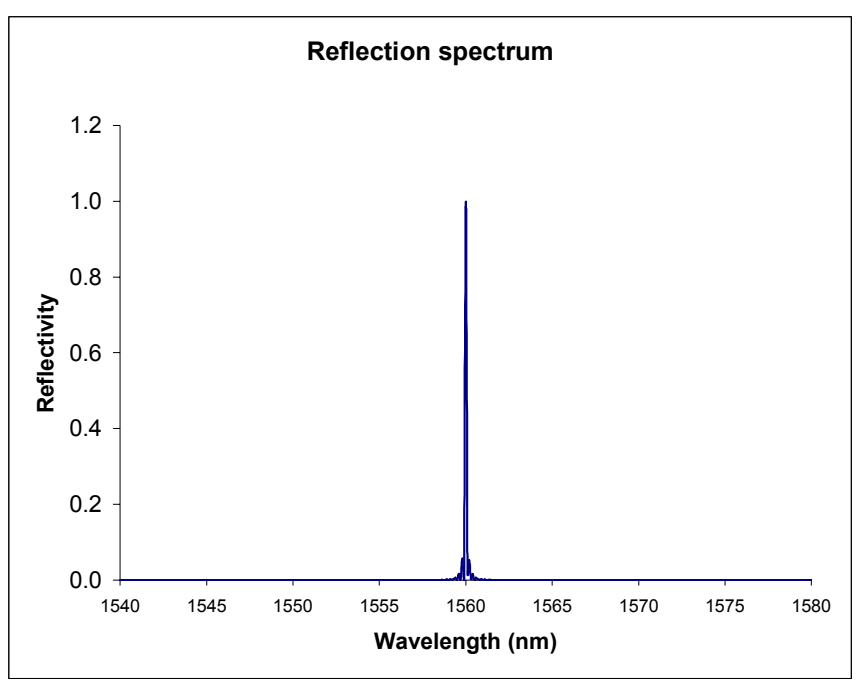

Figure 5 - Reflectivity spectrum of a solution for a Bragg grating designed by EPSO to filter the $1560 \mathrm{~nm}$ wavelength. Reflectivity is defined as the ratio between the amplitudes of the reflected and the incident waves.

\section{APPLICATION OF EPSO IN POWER SYSTEMS}

\subsection{Loss reduction: EPSO vs. Simulated Annealing}

The problem of loss reduction in power networks is related to finding a set of control actions that originate branch flows in such a way that power losses are minimized. Although the objective function is well defined, the control action set includes setting excitation levels at generators or taps at transformers and capacitor banks - this means that some variables may be continuous while other are of discrete nature. In a general form

$$
\begin{array}{ll}
\text { Minimize } & \mathrm{P}_{\text {losses }}(\mathrm{u}, \mathrm{x}) \\
\text { Subject } & \varphi(u, x, p)=0 \\
& \phi(u, x, p) \leq 0
\end{array}
$$

The first set of constraints describes the power flow equations and the second set is associated with operation limits, namely maximum branch flows and limits on admissible voltage levels.

We have compared the performance of EPSO with a Simulated Annealing model developed at INESC [12] which has been integrated in a DMS environment as is in use in some utilities. The test was conducted over the system defined in [13], with 24 nodes/36 branches, including 31 transmission lines, 5 transformers, 11 capacitor banks and 9 synchronous generators.

The test was conducted by manipulating only the discrete controls (transformer and capacitor bank taps). For both methods the stopping criterion has been defined as running 270 iterations without objective function improvement.

A typical convergence pattern observed for both algorithms can be observed in Figure 5.

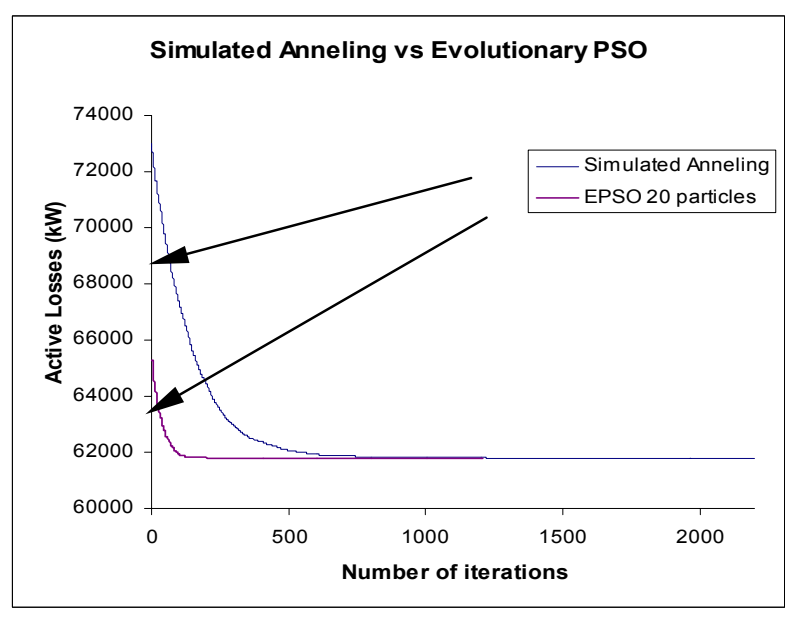

Figure 6 - Comparison between convergence in both EPSO and Simulated Annealing.

We found that EPSO not only finds better solutions but that it is a more robust algorithm. Both algorithms depend on random initialization and random numbers to progress; therefore it is normal that not always the same solution is reached. What is important is that a user may trust the algorithm, i.e., that it will always provide a near-optimal solution.

\begin{tabular}{||l|c|c||}
\hline \multicolumn{1}{|c|}{ Algorithm } & $\begin{array}{c}\text { Average losses } \\
(\mathrm{MW})\end{array}$ & $\begin{array}{c}\text { Mean sq. error } \\
(\mathrm{kW})\end{array}$ \\
\hline EPSO 10 particles & 61.7889 & 1.96607 \\
\hline EPSO 20 particles & 61.7880 & 1.48473 \\
\hline Simulated Annealing & 61.7921 & 9.81175 \\
\hline
\end{tabular}

As we can see in the table above, EPSO reveals superiority in terms of the solution found (evaluated by the average optimum obtained in 1000 runs) and in terms of its robustness (evaluated as the root of the mean square error relative to the best solution found).

In particular, EPSO gives consistently a near-optimum result, while the Simulated Annealing model failed many times to reach a solution as good (and that's why the dispersion of results in this case is much larger than with EPSO).

Therefore, EPSO is a much more reliable algorithm for practical applications.

\subsection{Voltage control}

For this test we've increase the load in several buses of the same IEEE 24-bus system. The voltage levels became very low and we run the EPSO algorithm to re-dispatch the 
reactive power in order to set the voltage back inside the limits, having as controls only the transformer taps. This scenario was designed in such a way that no solution would be found that would respect all the nodal voltage limits.

If no solution satisfies all constraints, some criterion must be specified in order to select some acceptable alternative. We have put EPSO/20 particles running under several criteria with success, and we will refer to two: a) minimizing the sum of voltage deviations outside the admissible voltage band; b) minimizing the maximum deviation relative to the admissible voltage band. Notice that the second criterion is again of the min-max type.

Figure 7 illustrates the two solutions obtained with the two criteria, departing from the same initial condition and specifying a lower voltage admissible limit of 0.95 p.u.. The fact to notice is that EPSO could work as well with a linear criterion and with a min-Max criterion and produce high quality solutions.

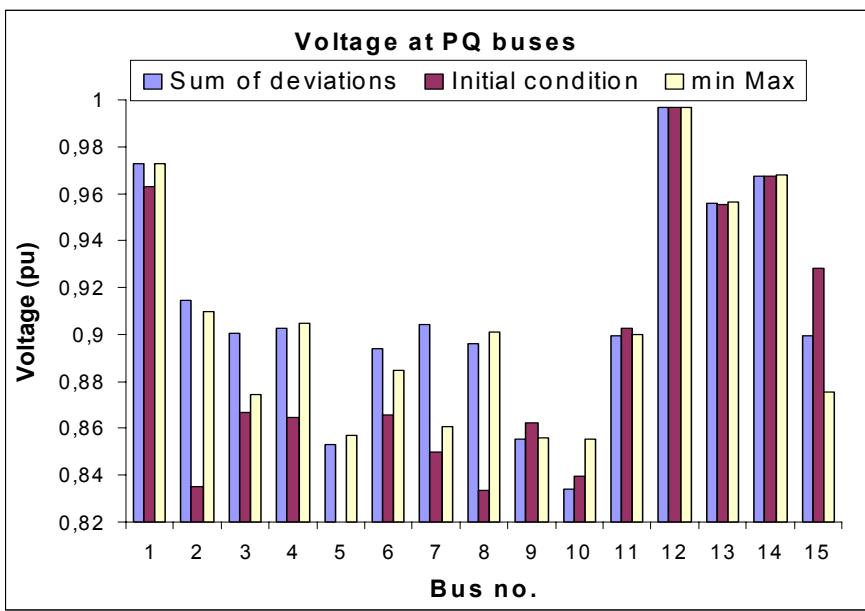

Figure 7 - Comparison of best alternatives under two criteria for nodal voltages in a problem with no feasible solution.

\section{CONCLUSIONS}

This papers reports a new and successful meta-heuristic built over the concepts of Evolution Strategies and Particle Swarm Optimization - EPSO, the Evolutionary self-adaptive Particle Swarm Optimization.

In a number of difficult test problems, EPSO proved its superiority over Classical PSO. And in Power System problems it showed superiority over Simulated Annealing.

EPSO not only found better solutions than the other methods in competition, but also showed to be a more reliable and robust method. It is largely independent of parameter initialization, due to its self-adaptive characteristics, and it is also largely independent of random initialization, showing a very small dispersion in results for a large number of independent runs for the same problem.

\section{REFERENCES}

[1] Rechenberg, I. (1973), “Evolutionsstrategie-Optimierung technischer Systeme nach Prinzipen der biologischen Evolution", Frommann-Holzboog, Stuttgart

[2] Schwefel, H.-P. (1995), "Evolution and Optimum Seeking”, Ed. Wiley, New York NY

[3] Fogel, D.B. (1992), "Evolving Artificial Intelligence", Ph.D. Thesis, University of California, San Diego

[4] Kennedy, J., Eberhart, R.C. (1995), "Particle Swarm Optimization", Proceedings of the 1995 IEEE International Conference on Neural Networks, pp. 19421948, Perth, Australia, 1995

[5] F. van den Bergh, A.P. Engelbrecht, "Training Product Unit Networks using Cooperative Particle Swarm Optimization", International Joint Conference on Neural Networks (IJCNN), Washington D.C., 2001

[6] Lovberg, M. et al. (2001), "Hybrid Particle Swarm Optimiser with Breeding and Sub-populations", Proceedings of GECCO2001 - Genetic and Evolutionary Computation Conference 2001(pp. 409), July 7-11 2001, San Francisco CA, USA

[7] Clerc, M. (1999), "The Swarm and the Queen: Towards a Deterministic and Adaptive Particle Swarm Optimization", Proceedings of the 1999 Congress of Evolutionary Computation, vol. 3, 1951-1957, IEEE Press

[8] Yuhui Shi, Russell C. Eberhart, "Parameter Selection in Particle Swarm Optimization", Proceedings of the Seventh Annual Conference on Evolutionary Programming, 1998

[9] Beyer, H.-G. (1996), "Toward a Theory of Evolution Strategies: Self-Adaptation", in Evolutionary Computation, vol. 3, no. 3, pag. 311-347

[10] Vladimiro Miranda, Nuno Fonseca, "EPSOEvolutionary self-adapting Particle Swarm optimization", internal report INESC Porto, July 2001 (obtainable from the authors by request)

[11] Vladimiro Miranda, Nuno Fonseca, "Design of optical Bragg grating filters with EPSO”, internal report INESC Porto, October 2001 (obtainable from the authors by request)

[12] Jorge Pereira, J. Tomé Saraiva, Maria Teresa Ponce de Leão, "Identification of Operation Strategies of Distribution Networks Using a Simulated Annealing Approach", Proceedings of IEEE Budapest Power Tech'99, paper BPT99-357-17, August 1999.

[13] Reliability Test System Task Force of the Application of Probability Methods Subcommittee, "IEEE Reliability Test System ", IEEE Trans. On Power Apparatus and Systems, vol. PAS-98, no. 6, Nov./Dec. 1979. 